\title{
Nonpenetrating Damage Identification Using Hybrid Lamb Wave Modes from Hilbert-Huang Spectrum in Thin-Walled Structures
}

\author{
Zijian Wang, ${ }^{1}$ Pizhong Qiao, ${ }^{2}$ and Binkai Shi ${ }^{3}$ \\ ${ }^{1}$ Department of Dam Safety Management, Nanjing Hydraulic Research Institute, Nanjing 210029, China \\ ${ }^{2}$ Department of Civil and Environmental Engineering and Composite Materials and Engineering Center, Washington State University, \\ Pullman, WA 99164-2910, USA \\ ${ }^{3}$ School of Mechanics and Materials, Hohai University, Nanjing 210098, China
}

Correspondence should be addressed to Zijian Wang; zijianwang@nhri.cn

Received 12 June 2017; Revised 19 September 2017; Accepted 16 October 2017; Published 20 November 2017

Academic Editor: Sandris Ručevskis

Copyright (C) 2017 Zijian Wang et al. This is an open access article distributed under the Creative Commons Attribution License, which permits unrestricted use, distribution, and reproduction in any medium, provided the original work is properly cited.

\begin{abstract}
Lamb waves have shown promising advantages for damage identification in thin-walled structures. Multiple modes of Lamb wave provide diverse sensitivities to different types of damage. To sufficiently utilize damage-related wave features, damage indices were developed by using hybrid Lamb wave modes from Hilbert-Huang spectra. Damage indices were defined as surface integrals of Hilbert-Huang spectra on featured regions determined by time and frequency windowing. The time windowing was performed according to individual propagation velocity of different Lamb wave mode, while the frequency windowing was performed according to the frequency of excitation. By summing damage indices for all transmitter-receiver pairs, pixels were calculated to reconstruct a damage map to characterize the degree of damage at each location on structure. Both numerical and experimental validations were conducted to identify a nonpenetrating damage. The results demonstrated that the proposed damage indices using hybrid Lamb wave modes are more sensitive and robust than the one using single Lamb wave mode.
\end{abstract}

\section{Introduction}

Applying walls as thin as possible is a natural optimization to minimize dead load and reduce construction cost. These thin-walled structures have been widely used in aircraft fuselages, storage vessels, sluices, and so forth. However, longperiod service deteriorates structural resistance and increases the possibility of failure. Impact, abrasion, and corrosion could cause nonpenetrating damage on thin-walled structures. It is imperative to identify this damage as early as possible to prevent it from penetrating the cross-section and causing catastrophic failure.

Recently, Lamb waves have shown promising suitability for damage identification in thin-walled structures. Irradiating through thickness and propagating over substantial areas, Lamb waves can assess structural integrity without probe movement. Due to high vibration frequency, typically from kilohertz to megahertz [1], Lamb waves are sensitive to small damage at millimeter scale, which are hard to be identified by low-frequency methods. Furthermore, multiple modes of Lamb wave provide diverse sensitivities to identify different types of damage. Among all the Lamb wave modes, fundamental symmetric $S_{0}$ [2] and antisymmetric $A_{0}$ [3] modes have been widely used because of their great amplitude. Based on the dependence of amplitude on frequency of excitation and propagation velocity, we can intentionally amplify or depress Lamb wave modes to obtain a wave field dominated by single mode. Many researches have been reported to separate Lamb wave mode [4-6] and use single mode to identify damage. Using single Lamb wave mode with nondispersive velocity and low attenuation [7], various kinds of damage, including crack [8], delamination [9], 


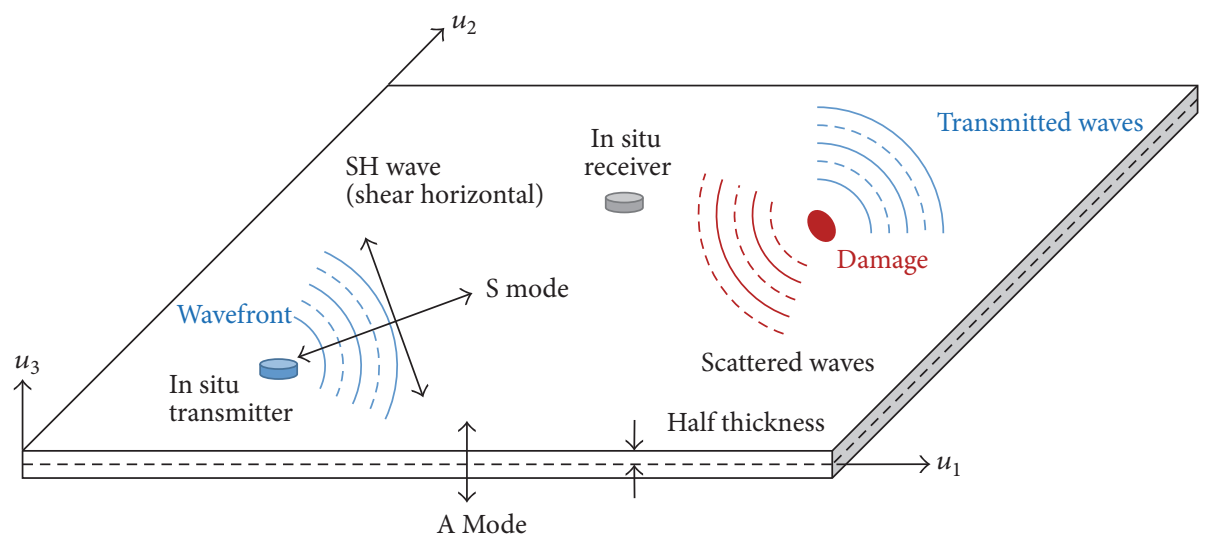

FIGURE 1: Propagating waves in thin-walled structures.

distributed micro defect [10], fatigue [11], and corrosion [12], have been identified. Various damage indices have been developed to interpret Lamb wave signals and alleviate noises by transforming Lamb wave signals to different physical domains. The ways to transform Lamb wave signals include short-time Fourier [13], wavelet [14], and Hilbert-Huang [15] transformations. By plotting damage indices at each location, we can produce a damage map to characterize the degree of damage over structure.

However, using single Lamb wave mode neglects damagerelated features of other Lamb wave modes and, accordingly, compromises the identification of nonpenetrating damage. This deficiency becomes serious when adopting sparse sensors to identify nonpenetrating damage in noisy environment. To this end, it is desirable to use hybrid Lamb wave modes to enhance the sensitivity and robustness of sparse sensors to identify nonpenetrating damage. In this study, since there is no conclusive superiority of a specific Lamb wave mode to identify nonpenetrating damage, we used both $\mathrm{S}_{0}$ and $\mathrm{A}_{0}$ Lamb wave modes to develop damage indices. By transforming Lamb wave signals to a time-frequency spectrum via Hilbert-Huang transformation, damage indices were defined as surface integrals on featured regions determined by time and frequency windowing. The time windowing was performed according to individual propagation velocity of different Lamb wave mode, while the frequency windowing was performed according to the frequency of excitation. The usage of hybrid Lamb wave modes increased the capability of sparse sensors to identify nonpenetrating damage in noisy environment. Both numerical and experimental implementation presented damage more distinctly on damage map, indicating good improvement of the proposed method over the one using single mode.

\section{Wave Propagation in Thin-Walled Structures}

Wave propagation in thin-walled structures, for example, plates and shells, is guided by upper and bottom surfaces, producing Lamb waves in $u_{1}-u_{3}$ plane, and shear horizontal

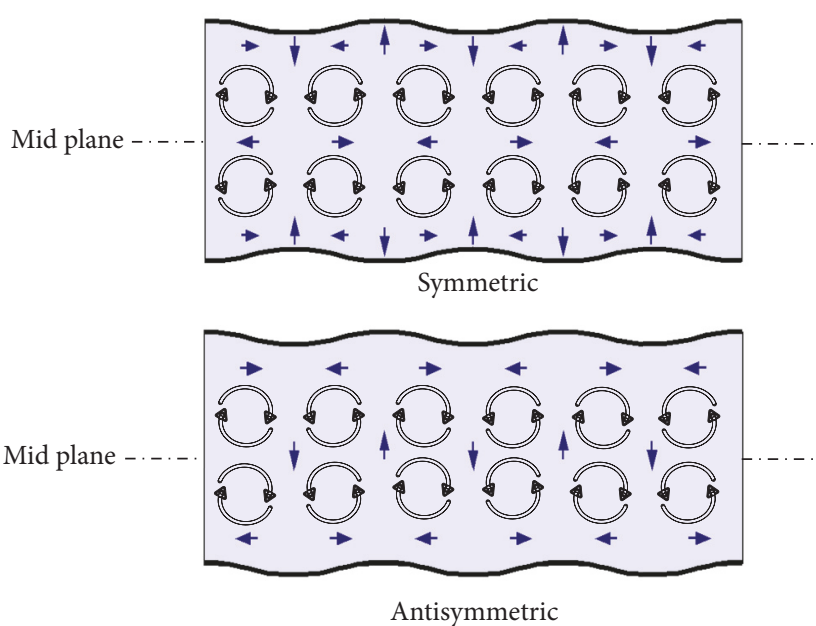

FIGURE 2: Displacement of Lamb wave modes.

(SH) wave in $u_{1}-u_{2}$ plane (see Figure 1). According to the displacement pattern, Lamb waves can be seen as symmetric and antisymmetric modes (see Figure 2). For the application of Lamb waves on damage identification, we use group velocity to characterize wave propagations. The group velocity of $\mathrm{SH}$ wave is solved as the velocity of shear vertical wave $c_{T}$ [16], while the group velocities of Lamb waves are solved from the Rayleigh-Lamb equation [17]:

$$
\begin{aligned}
\frac{\tan p h}{\tan q h} & =-\left[\frac{4 k^{2} p q}{\left(k^{2}-q^{2}\right)^{2}}\right]^{ \pm 1} \\
p & =\sqrt{\left(\frac{\omega}{c_{L}}\right)^{2}-(k)^{2}} \\
q & =\sqrt{\left(\frac{\omega}{c_{T}}\right)^{2}-k^{2}},
\end{aligned}
$$

where $h$ is the half thickness of plate; $k$ is the wave number; $\omega$ is the circular frequency; $c_{L}$ is the velocity of longitudinal 


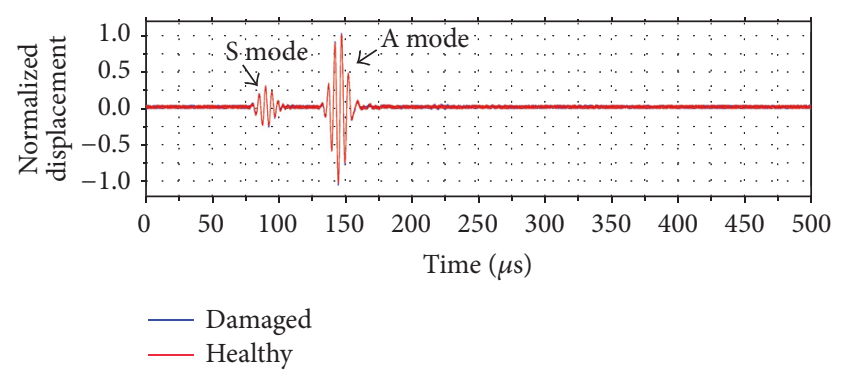

FIGURE 3: Lamb wave signals.

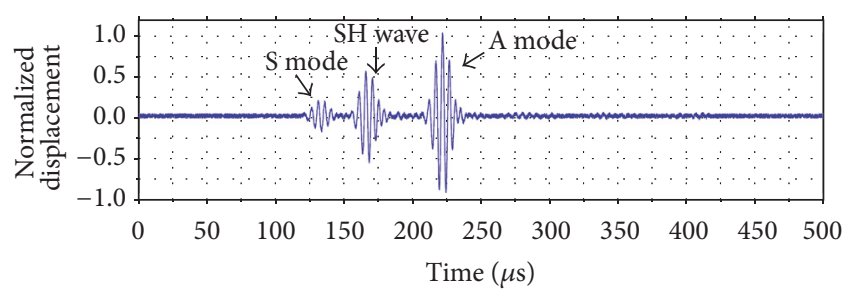

FIGURE 4: Residual signal.

wave; $c_{T}$ is the velocity of shear vertical wave; and +1 and -1 represent symmetric and antisymmetric modes, respectively.

\section{Physical Process of Damage Identification}

Figure 3 shows two Lamb signals acquired from healthy and damaged finite element (FE) models, respectively. Details about the FE model are given in Section 6. Two wave peaks around 90 and $140 \mu$ s correspond to $S_{0}$ and $A_{0}$ Lamb wave modes, respectively. Amplitudes of different Lamb wave modes can be controlled by changing the tuning frequency of excitation [18]. In this case, $A_{0}$ mode gains triple amplitude as $\mathrm{S}_{0}$ mode. Since the damaged signal contains direct waves from the excitation and scattered waves from damage, there is small difference between healthy and damaged signals, as shown in Figure 3. To extract scattered waves caused by damage, we subtract healthy signal from damaged one to get a residual signal, as shown in Figure 4. Since boundary reflections are the same for damaged and healthy signals, this subtraction can eliminate the interference of boundary reflection, and the overlap of direct waves and boundary reflections cannot affect the residual signal. At this point, we could see the residual signal as a wave transmitted by damage as a second wave source. Since different waves propagate with individual velocities, we can obtain separated Lamb wave modes as different wave peaks in the residual signal by deploying sensors at a distance from the damage.

In this case, mode conversion at damage generates $\mathrm{SH}$ waves in the residual signal along with $S_{0}$ and $A_{0}$ modes. The phases of these wave peaks indicate the location of damage. By multiplying time interval between excitation and these wave peaks with wave velocity, we can determine the distance from transmitter through damage to receiver and draw an ellipse on which possible damage locates (see Figure 5). The superposition of multiple ellipses determined by each transmitter-receiver pair finally presents the location of damage.

\section{Hilbert-Huang Transformation}

For real situation, wave peaks in residual signal are inadequate to characterize damage because of their vulnerability to noise and insensitivity to weak damage scattering (e.g., scattered waves caused by nonpenetrating damage). In most cases, we need to develop damage indices from multiple physical domains to characterize damage. Therefore, HilbertHuang transformation (HHT) gains its popularity to analyze Lamb wave signals in time and frequency domains. The HHT provides intuitive and direct basis to characterize nonstationary Lamb wave signals based on a simple but reasonable assumption: a signal can be decomposed into a finite and a small number of simple oscillatory modes, with significantly different frequencies, one superimposed on another [19]. This assumption is suitable with real situation, since frequency of Lamb wave signal is significantly higher than environmental noises. These simple oscillatory modes are defined as intrinsic mode functions (IMFs) which satisfy two requirements: the number of extreme values and zero points must be equal or differ at most by one; and the function must be symmetric about the local mean. The IMFs are obtained by a sifting process. The local maxima and minima points of input signal are firstly identified to form an upper and lower envelope together with the mean curve $m_{1}(t)$. The difference between input signal $x(t)$ and mean curve $m_{1}(t)$ is designated as a prototype IMF $h_{1}(t)$. However, the prototype cannot meet the requirements of IMF in most cases, so the sifting process is repeated by treating the prototype IMF as another input signal through the above steps. This repetition 


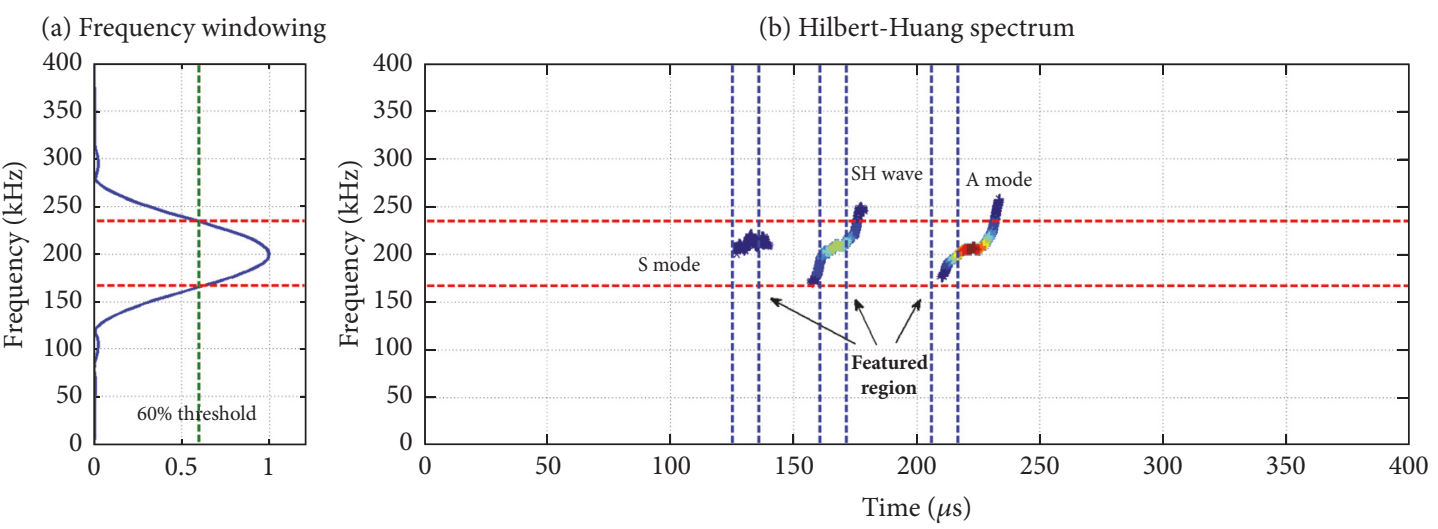

(c) Time windowing

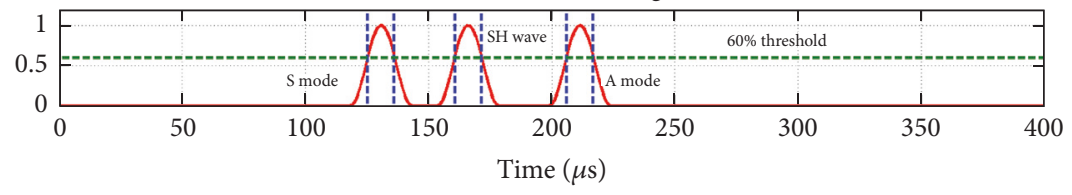

FIGURE 6: Formation of damage index.

aims to eliminate the low-frequency tendency and makes the signal more symmetric to meet the requirements of IMF. This sifting process stops when index $S D_{k}$ is smaller than a predetermined value as follows:

$$
S D_{k}=\frac{\sum_{t=0}^{T}\left|h_{k-1}(t)-h_{k}(t)\right|^{2}}{\sum_{t=0}^{T} h_{k-1}^{2}(t)},
$$

where $T$ is the sampling period. After $k$ times sifts, the prototype IMF $h_{1 k}(t)$ becomes the first IMF $c_{1}(t)$, which represents highest-frequency oscillation in input signal $x(t)$, whereas the residual $r_{1}(t)=x(t)-c_{1}(t)$ contains lowerfrequency oscillation. Then the residual $r_{1}(t)$ is treated as another input signal repeatedly in sifting process to obtain successive IMFs of lower frequency, until it becomes a constant or monotonic function, presenting original input signal $x(t)$ as follows:

$$
x(t)=\sum_{j=1}^{n} c_{j}(t)+r(t),
$$

where $c_{j}(t)$ is the $j$ th IMF and $r(t)$ is a final constant or a monotonic function.

The IMFs generated by this sifting process lead to a robust Hilbert transformation without the presence of negative frequencies. The Hilbert-Huang spectrum of the input signal $x(t)$ is obtained by summing the Hilbert spectrum of each IMF as follows:

$$
H(t, f)=\sum_{j=1}^{n} a_{j}(t) e^{i \int \omega_{i}(t) d t},
$$

where $a_{j}(t)$ and $\omega_{j}(t)$ are the instantaneous amplitude and frequency of the $j$ th IMF, respectively. At this point, the input signal $x(t)$ is represented as distinct energy distribution in a time-frequency spectrum where a damage index can be developed.

\section{Formulation of Damage Index}

Applying Hilbert-Huang transformation on the Lamb wave signal presented in Figure 4, a time-frequency spectrum is produced in Figure 6. The damage index is formulated by integrating the Hilbert-Huang spectrum on featured regions selected by time and frequency windowing.

For the frequency windowing, damage-related waves are assumed to have the same frequency with excitation. The width of frequency window is determined by a $60 \%$ threshold of the amplitude in Fast-Fourier spectrum of excitation (see Figure 6(a)). This threshold is chosen empirically to obtain a narrow frequency window to eliminate interferences from other frequencies and secure sufficient energy to develop robust damage indices.

For the time windowing, scattered waves are assumed to have the same waveform with excitation. At a given location $(x, y)$, the time-of-flight $\Delta t$ for a specific wave mode is estimated by

$$
=\frac{\sqrt{\left(x_{t}-x\right)^{2}+\left(y_{t}-y\right)^{2}}+\sqrt{\left(x_{r}-x\right)^{2}+\left(y_{r}-y\right)^{2}}}{v},
$$

where $x_{t}, y_{t}, x_{r}$, and $y_{r}$ are the coordinates of transmitter and receiver, respectively; and $v$ is the propagation velocity of a specific wave mode. By shifting envelop of excitation according to the time-of-flight calculated in (5), the envelope of wave peaks in residual signal can be estimated. The estimated time of arrival of $\mathrm{S}_{0}, \mathrm{SH}$, and $\mathrm{A}_{0}$ wave modes is shown in Figure 6(c). The envelop $y(t)$ is calculated by Hilbert transformation as follows:

$$
\begin{aligned}
H[x(t)] & =\frac{1}{\pi} P \int_{-\infty}^{\infty} \frac{x(\tau)}{t-\tau} d \tau \\
y(t) & =x(t)+i H[x(t)],
\end{aligned}
$$




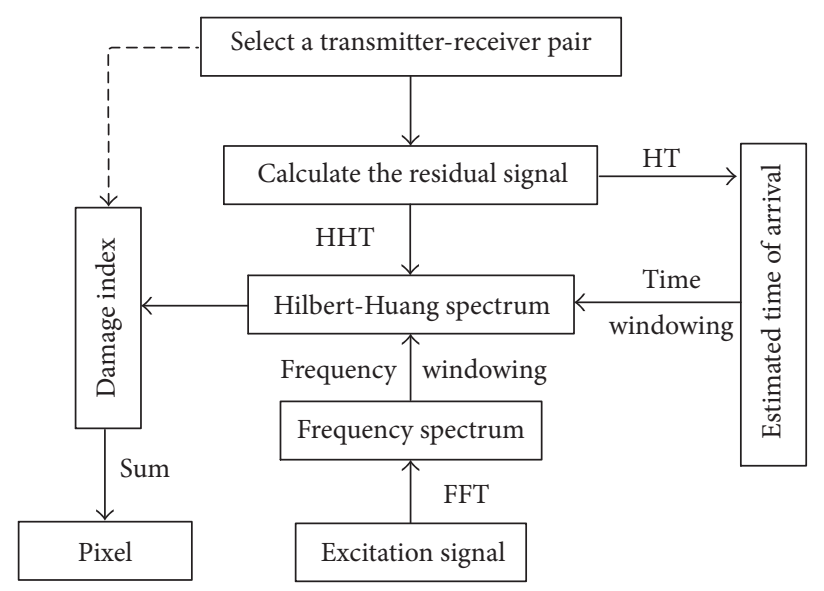

FIGURE 7: Procedure of damage identification.

where $P$ is the Cauchy principal value. The width of time window is determined by a $60 \%$ threshold of the envelop of excitation $|y(t)|$ (see Figure 6(c)). This threshold is chosen empirically to obtain a narrow time window to eliminate interferences from other wave modes and secure sufficient energy to develop robust damage indices.

After the time and frequency windowing, three rectangular featured regions are shown in Figure 6(b), associated with $\mathrm{S}_{0}, \mathrm{SH}$, and $\mathrm{A}_{0}$ wave modes, respectively. A damage index $\operatorname{DI}(x, y)$ is defined as surface integral of Hilbert-Huang spectrum on these featured regions as follows:

$$
\begin{aligned}
\operatorname{DI}(x, y)= & \iint_{\mathrm{S}_{0}} \operatorname{HHS}(t, f) d t d f \\
& +\iint_{\mathrm{A}_{0}} \operatorname{HHS}(t, f) d t d f \\
& +\iint_{\mathrm{SH}} \operatorname{HHS}(t, f) d t d f .
\end{aligned}
$$

The pixel at location $(x, y)$ on the damage map is defined as the sum of damage index determined by individual transmitter-receiver pair as follows:

$$
P(x, y)=\sum_{i=1}^{n} \mathrm{DI}_{i},
$$

where $i$ denotes the $i$ th transmitter-receiver pair.

At this point, damage is identified on a reconstructed map based on the proposed damage indices, by following the procedure, as shown in Figure 7. Pixels on the damage map indicate the degree of damage at this location.

\section{Numerical Implementation}

To validate the effectiveness of proposed damage indices, we simulated the physical process of wave excitation, propagation, and scattering in Abaqus 6.11. The FE model was in
$400 \times 400 \times 4$ (length $\times$ width $\times$ depth $) \mathrm{mm}$, discretized by $1 \times 1 \times 1 \mathrm{~mm}$ C3D8R elements. Damping elements were assigned around the plate to absorb boundary reflections. Four piezoelectric transducers (PZTs) were placed at corners of an AL6061-T6 aluminum plate to excite or receive Lamb waves. The excitation $(200 \mathrm{kHz}$ tone burst) was applied as nodal force on a rectangular region $(5 \times 5 \mathrm{~mm})$ in $x$-y plane (see Figure 8). Considering numerical stability and efficiency, time increment $\Delta t$ and duration were chosen as $1 \times 10^{-7} \mathrm{~s}$ and $1 \mathrm{~ms}$, respectively. Twelve elements were deleted from the mesh to simulate a $2 \times 2 \times 3$ (length $\times$ width $\times$ depth) $\mathrm{mm}$ nonpenetrating damage (see Figure 9). The coordinates of transducers and damage are shown in Figure 8, according to the Cartesian coordinate at plate center.

By exciting transducers in turns, Lamb waves were acquired as magnitude of displacements at receivers (see Figure 9), producing 16 signals in healthy and damaged plate, respectively. Transmitted and scattered waves were denoted in a snapshot, as shown in Figure 9. Scattered waves were extracted as residual signals by subtracting healthy signals from damaged ones (see Figure 10). However, mode conversion led to the presence of $\mathrm{SH}$ waves as second wave peak in residual signals, along with $S_{0}$ and $A_{0}$ Lamb wave mode as first and third wave peaks. Based on the velocity measurements on healthy plate, the group velocities of the $\mathrm{S}_{0}, \mathrm{~A}_{0}$, and $\mathrm{SH}$ waves were determined as 5183,2982 , and $3892 \mathrm{~m} / \mathrm{s}$, respectively. Since scattered waves were affected by incident angles and shape of damage, it is difficult to obtain comparative amplitude for different scattered waves.

To examine noisy immunity of the proposed damage indices, $150 \%$ Gaussian noises were blended into residual signals. Following the procedure of damage identification in Figure 7 , normalized damage maps were produced using $S_{0}$ mode, $\mathrm{A}_{0}$ mode, $\mathrm{SH}$ wave, and hybrid waves, respectively (see Figure 11). Pixels on these damage maps were calculated according to (8). Large pixel indicates strong wave scattering, which relates to the presence of damage. The damage maps using single wave mode are interfered by Gaussian noises, 

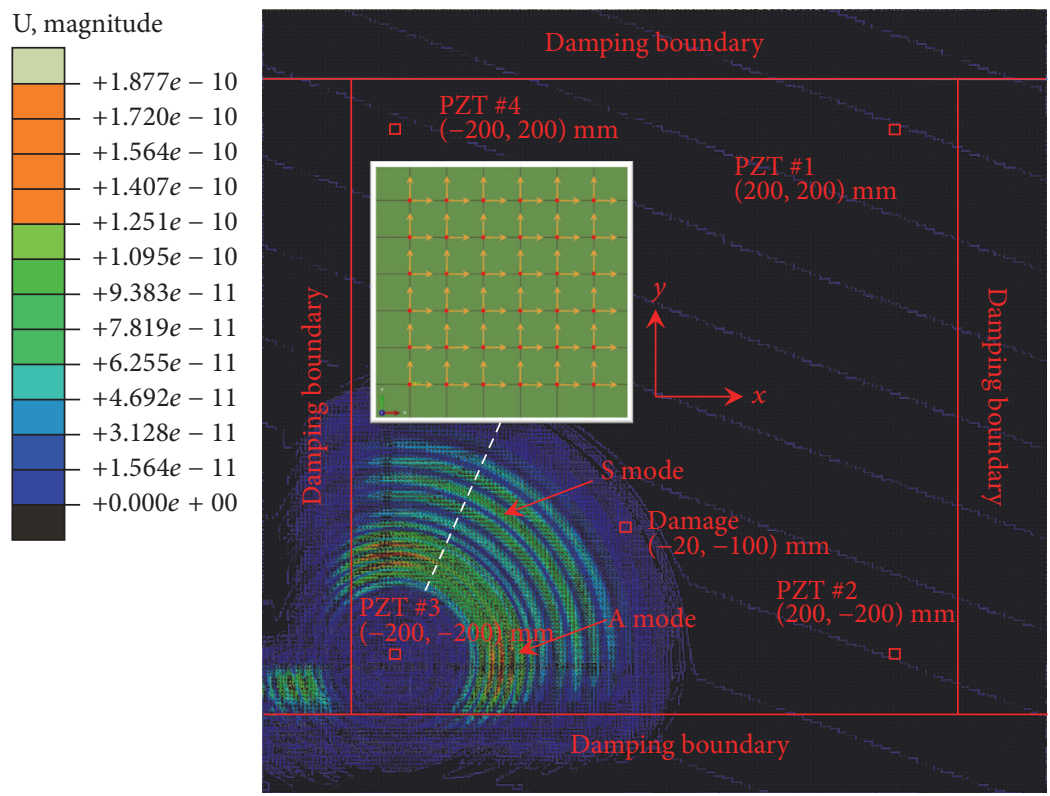

Figure 8: Wave excitation in FE model.
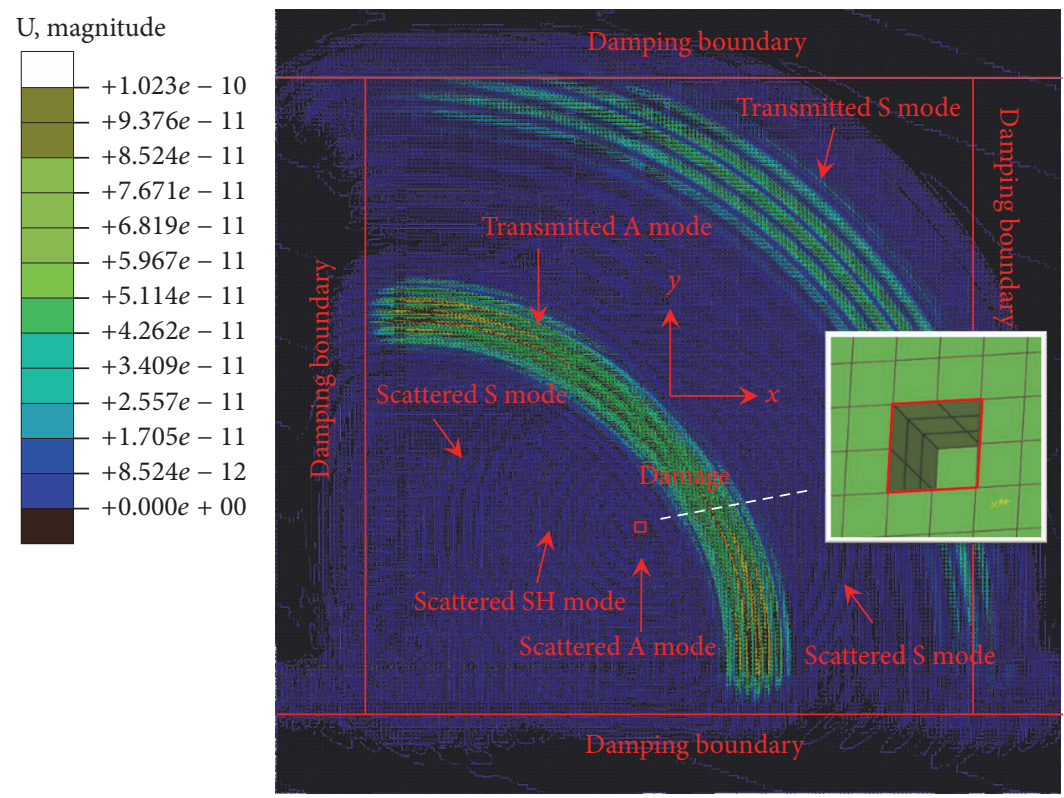

FIgURE 9: Wave scattering at damage.

presenting a blurry damage identification at the location of $(-20,-100 \mathrm{~mm})$, along with a lot of false damage spots (red pixel) at other places, while the damage map using hybrid wave modes obtains appropriate amplification of pixel at the location of $(-20,-100 \mathrm{~mm})$, presenting a concentrated damage identification. The comparison between these damage maps indicates that the proposed damage indices are more sensitive and robust to identify nonpenetrating damage in noisy environment than the ones using single wave mode.

\section{Experimental Implementation}

In parallel, we conducted an experiment to identify a nonpenetrating damage in an AL6061-T6 aluminum plate, whose dimensions were $700 \times 800 \times 3$ (length $\times$ width $\times$ depth) $\mathrm{mm}$. Four PZT disks ( $5 \mathrm{~mm}$ in diameter, $0.5 \mathrm{~mm}$ in thickness) were surface-bonded by epoxy at corners (see Figure 12). A nonpenetrating damage was manufactured by drilling a groove in size of $4 \times 4 \times 1$ (length $\times$ width $\times$ depth) $\mathrm{mm}$. 

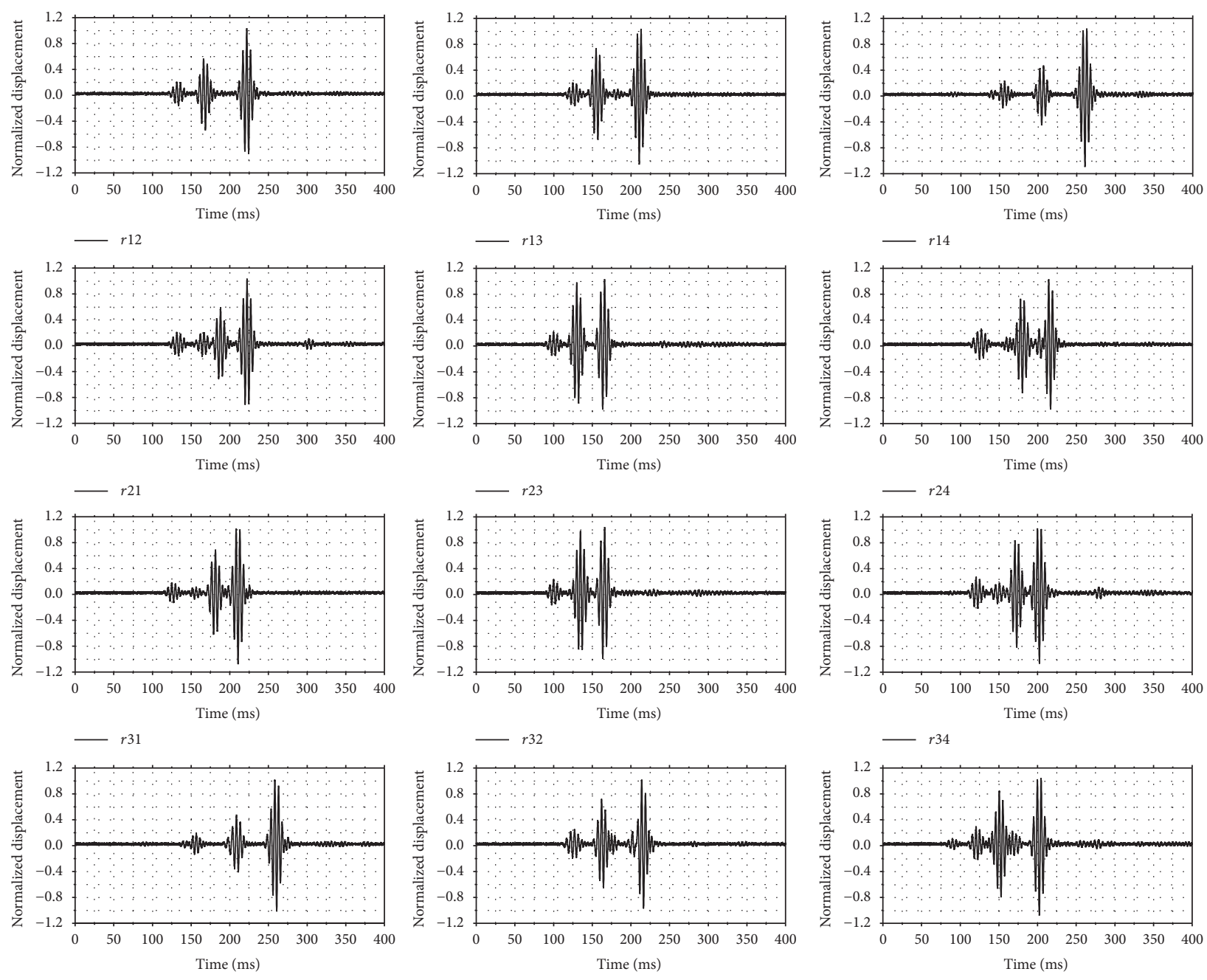

FIGURE 10: Residual signal of different transmitter-receiver pair (FE simulation).

A $190 \mathrm{kHz}$ tone burst was generated by an Agilent 3325A waveform generator. The voltage of tone burst was amplified to $200 \mathrm{Vpp}$ by a PINTEK HA-405 voltage amplifier. A signal that came from monitoring terminal of the voltage amplifier was imported to an Agilent DSO7034B digital oscilloscope as trigger to coordinate excitation and reception. Once a transmitter excited, the oscilloscope recorded voltages of receivers in $1.7 \mathrm{~ms}$ with a sampling frequency of $10 \mathrm{MHz}$ (see Figure 13). Since the circular groove scattered waves in all directions evenly, mode conversion at circular groove was not as serious as rectangular damage shown in the numerical FE simulation in Section 6. Only $\mathrm{S}_{0}$ and $\mathrm{A}_{0}$ Lamb wave modes were clearly presented, with group velocities of 4822 and $2993 \mathrm{~m} / \mathrm{s}$, respectively. Accordingly, damage indices in the following damage mapping adopted the first two terms in (7). Received signals were filtered by a Krohn-Hite 3384 filter (band pass from 180 to $200 \mathrm{kHz}$ ) and then blended with $100 \%$ Gaussian noises. By calculating pixels according to (8), damage maps reconstructed by $\mathrm{S}_{0}$ mode, $\mathrm{A}_{0}$ mode, and hybrid
Lamb wave modes are shown in Figure 14. As presented in the numerical implementation, the proposed damage indices are more sensitive and robust to nonpenetrating damage in noisy environment than the indices using single wave mode. In Figure 14(c), pixels near actual location of the damage (black dot) are amplified by hybrid Lamb wave modes, while random noises are offset by each other, producing small pixels at other places.

\section{Discussion and Conclusions}

In this study, damage indices using hybrid Lamb wave modes were proposed, and they were successively implemented in both numerical and experimental cases. Residual signals generated by transmitter-receiver pairs were calculated by subtracting healthy signals from damaged ones. The subsequent, residual signals were subjected to Hilbert-Huang transformation to produce time-frequency spectra. By windowing Hilbert-Huang spectra from time and frequency 


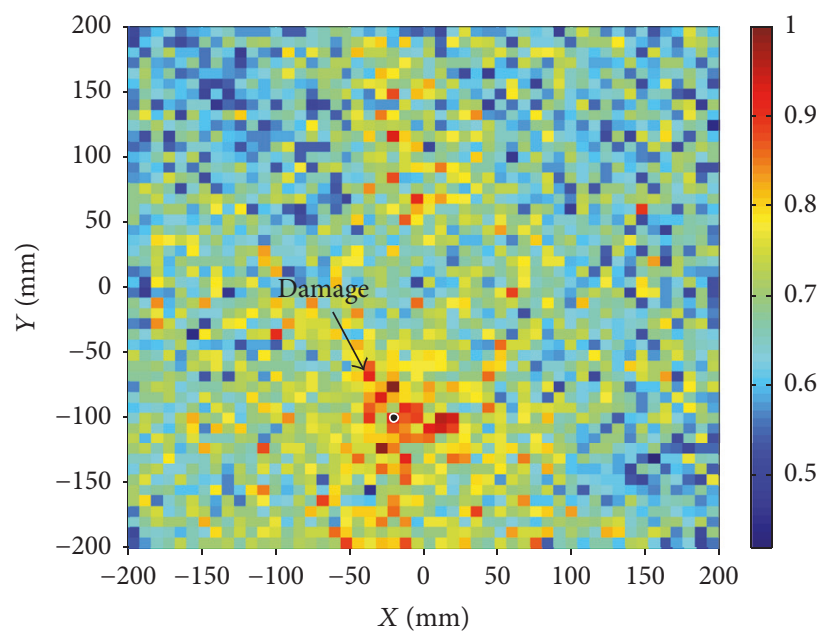

(a) $S_{0}$ wave only

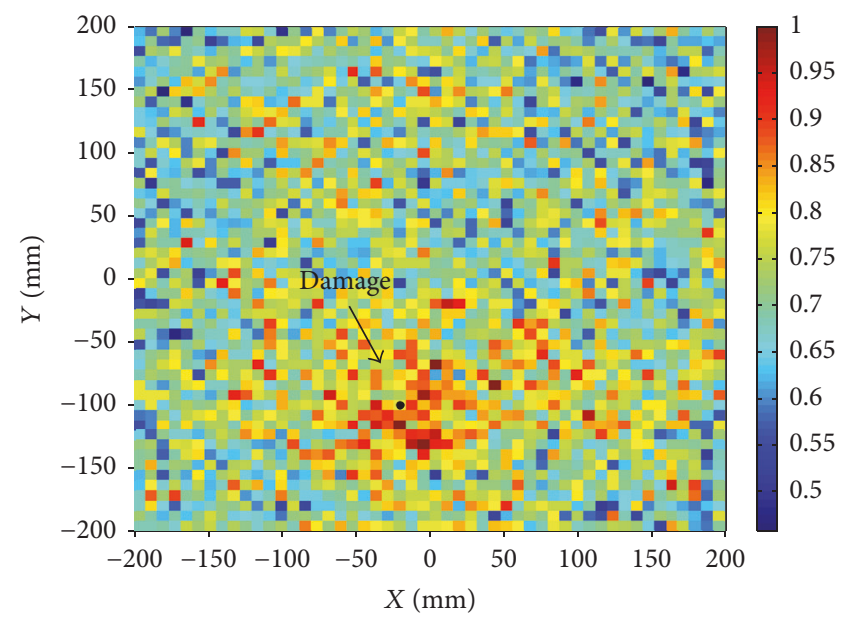

(c) SH wave only

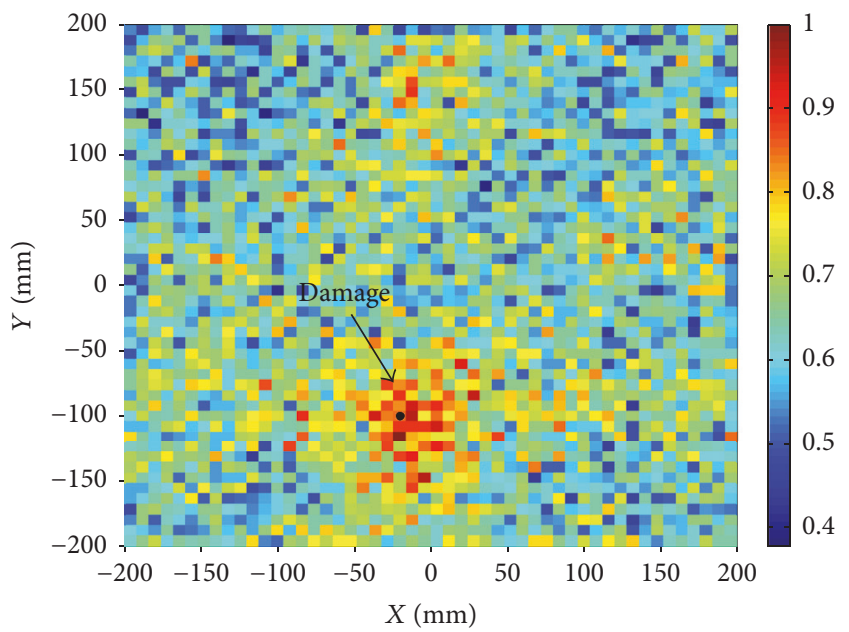

(b) $A_{0}$ wave only

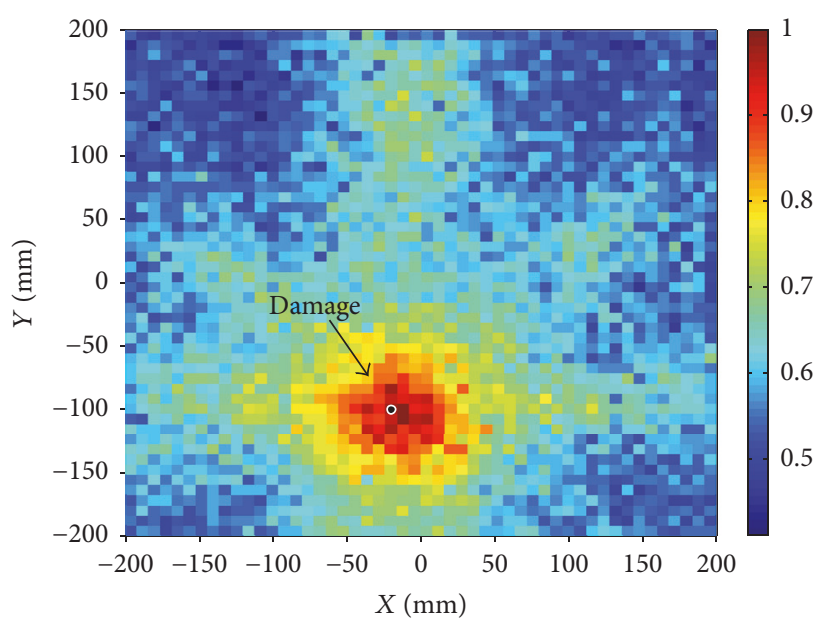

(d) Hybrid wave modes

FIGURE 11: Comparison of damage maps (FE simulation).

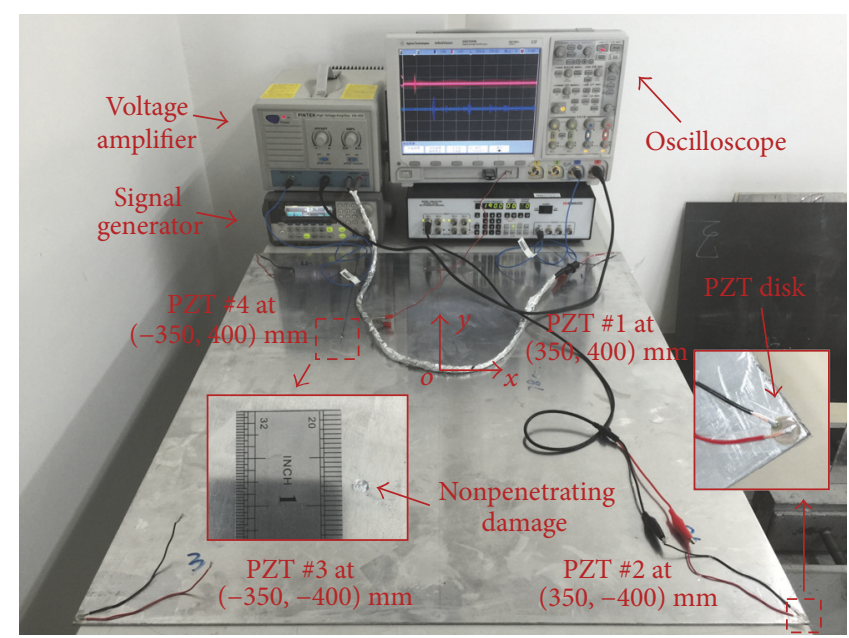

FIGURE 12: Experiment setup. 

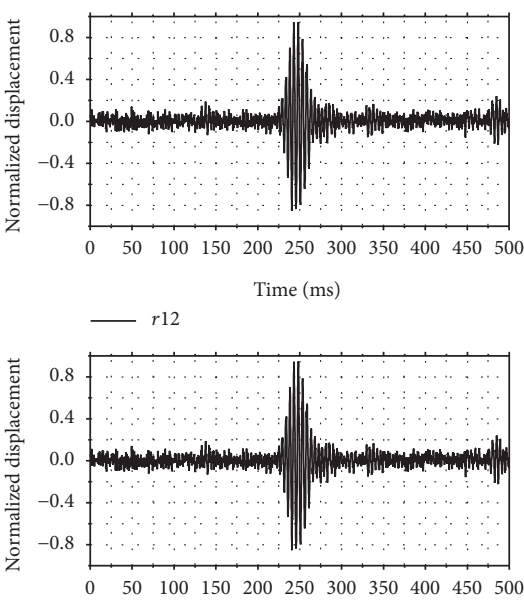

Time (ms)
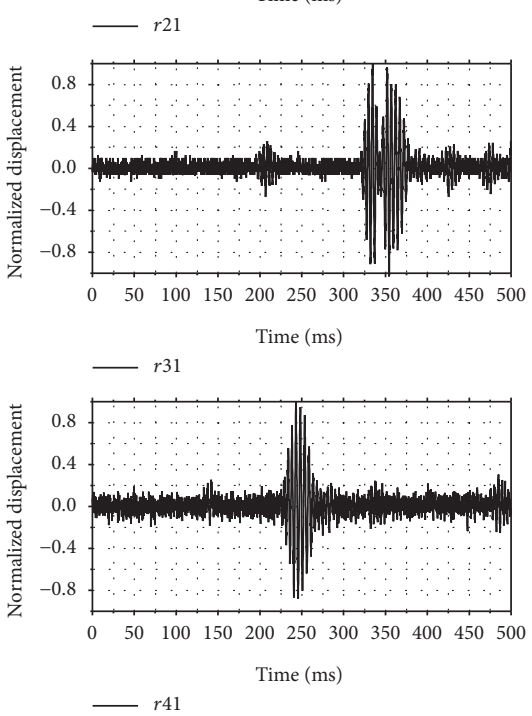

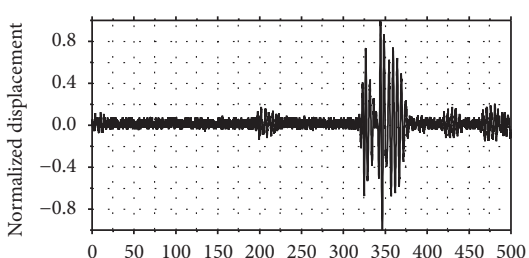

Time (ms)

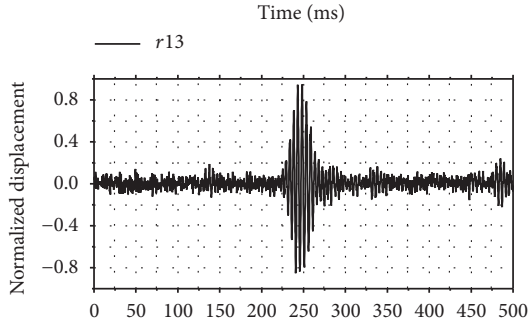

Time (ms)

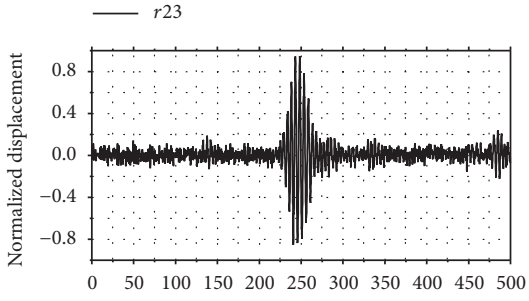

Time (ms)

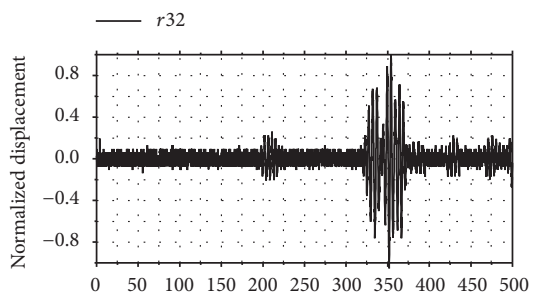

Time (ms)

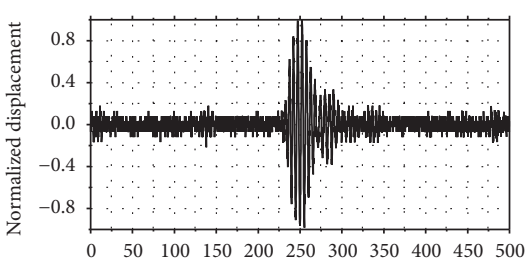

Time (ms)

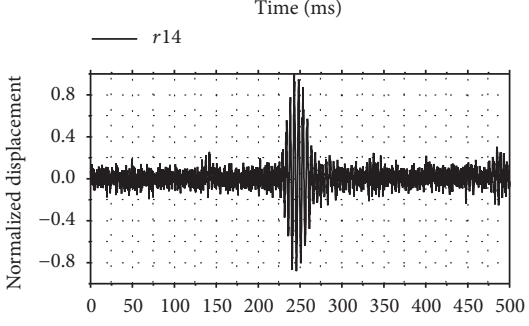

Time (ms)

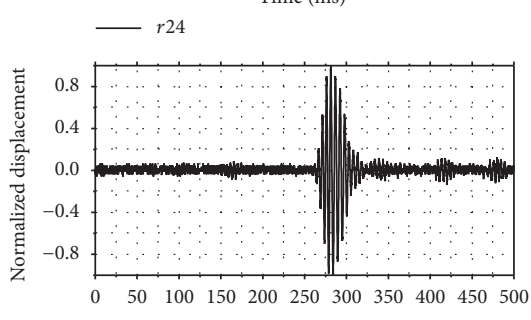

Time (ms)

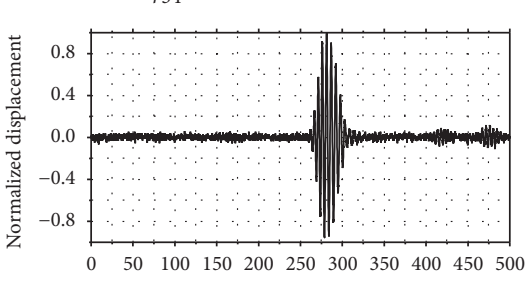

Time (ms)

FIGURE 13: Residual signals of different transmitter-receiver pair (experiment).

domains, featured regions were determined where the damage indices were formulated. The time windowing was performed according to individual propagation velocity of different Lamb wave modes, while the frequency windowing was performed according to the Fourier spectrum of excitation. The damage index was defined as surface integrals of HilbertHuang spectrum on these featured regions. By summing damage indices produced by all transmitter-receiver pairs, we reconstructed a damage map whose pixels indicate the degree of damage at each location on the plate. As shown in both numerical and experimental implementations, the usage of hybrid Lamb wave modes increases the signal-to-noise ratio, and it consequently improves sensitivity and robustness to nonpenetrating damage.

With successful damage identification before penetrating through wall cross-section, as presented in this study, we are able to warn about potential failure at early stage and, accordingly, improve the reliability of thin-walled structures. Moreover, the implementation of in situ transducers, as shown in the experiment, facilitates real-time structural health monitoring instead of periodical inspection. However, insufficient consideration of wave scattering at damage leads to varying amplitude for different scattered waves, which compromised the improvement of hybrid wave method in some degree. It is encouraging to study the amplitude changes and mode conversion of scattered waves in the future research.

\section{Conflicts of Interest}

The authors declare that they have no conflicts of interest.

\section{Acknowledgments}

The first author wants to acknowledge the financial support received from the National Key Research and Development Program of China (Grant no. 2016YFC0401603) and the Fund 


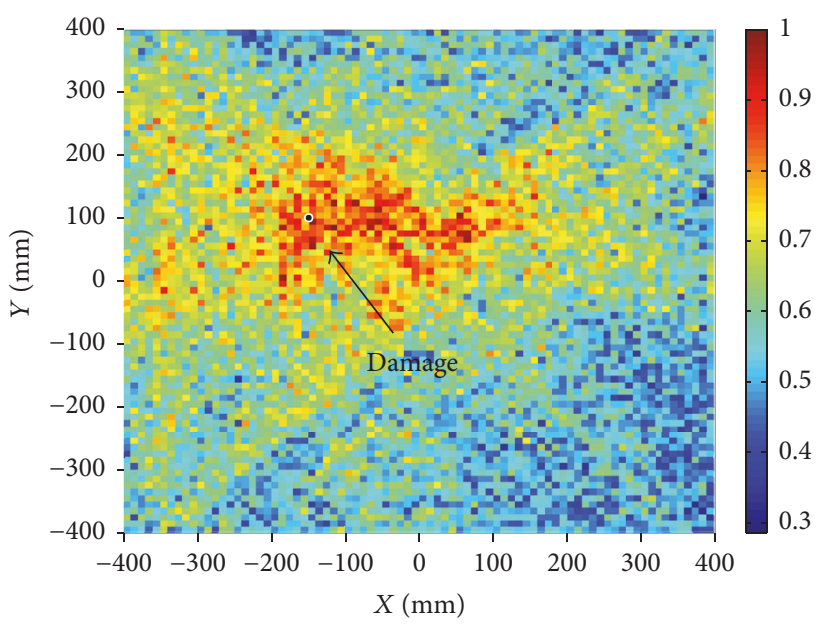

(a) $S_{0}$ wave only

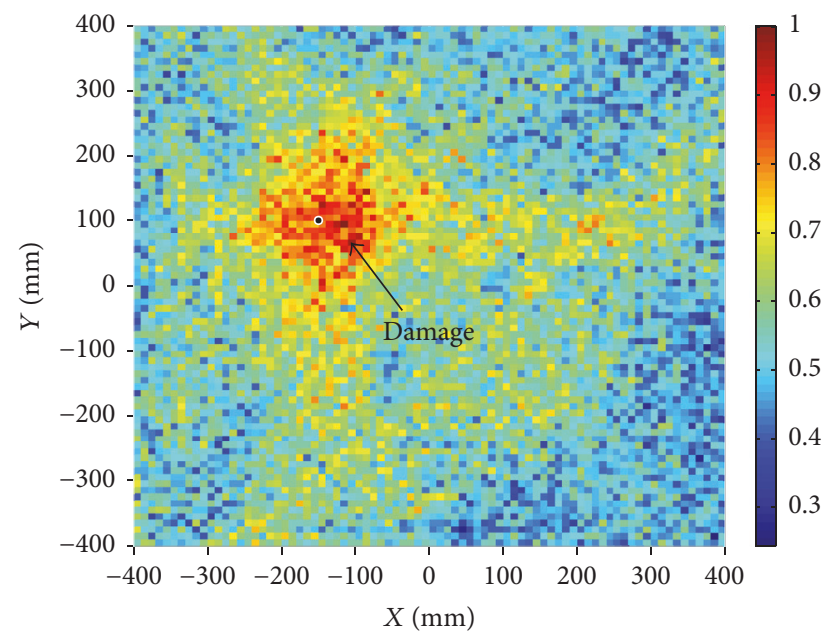

(b) $\mathrm{A}_{0}$ wave only

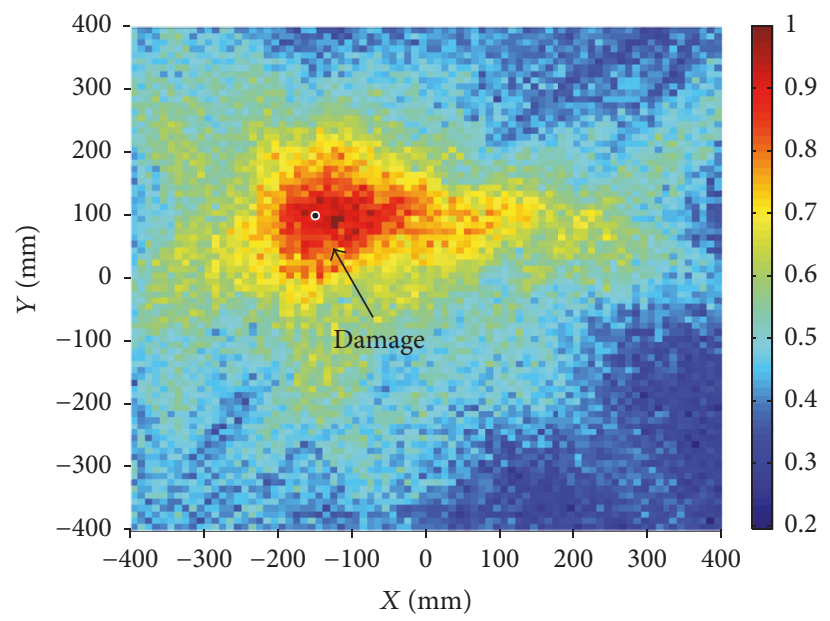

(c) Hybrid Lamb wave modes

FIGURE 14: Comparison of damage maps (experiment).

of Nanjing Hydraulic Research Institute (Grant no. Y716021) to this study.

\section{References}

[1] S. Gopalakrishnan, M. Ruzzene, and S. Hanagud, "Signal Processing Techniques," in Computational Techniques for Structural Health Monitoring, Springer Series in Reliability Engineering, pp. 97-154, Springer London, London, 2011.

[2] Z. Wang, P. Qiao, and B. Shi, "Application of soft-thresholding on the decomposed Lamb wave signals for damage detection of plate-like structures," Measurement, vol. 88, pp. 417-427, 2016.

[3] N. Quaegebeur, P. Micheau, P. Masson, and A. Maslouhi, "Structural health monitoring strategy for detection of interlaminar delamination in composite plates," Smart Materials and Structures, vol. 19, no. 8, Article ID 085005, 2010.

[4] I. Park, Y. Jun, and U. Lee, "Lamb wave mode decomposition for structural health monitoring," Wave Motion, vol. 51, no. 2, pp. 335-347, 2014.

[5] L. Zeng, J. Lin, J. Bao, R. P. Joseph, and L. Huang, "Spatial resolution improvement for Lamb wave-based damage detection using frequency dependency compensation," Journal of Sound and Vibration, vol. 394, pp. 130-145, 2017.

[6] M. Ratassepp, Z. Fan, and K. Lasn, "Wave mode extraction from multimodal wave signals in an orthotropic composite plate," Ultrasonics, vol. 71, pp. 223-230, 2016.

[7] K. Xu, D. Ta, P. Moilanen, and W. Wang, "Mode separation of Lamb waves based on dispersion compensation method," The Journal of the Acoustical Society of America, vol. 131, no. 4, pp. 2714-2722, 2012.

[8] Z. Wang and P. Qiao, "Backward wave separation method in a single transmitter and multi-receiver sensor array for improved damage identification of two-dimensional structures," International Journal of Damage Mechanics, vol. 26, no. 2, pp. 229-250, 2017.

[9] X. Yu, M. Ratassepp, and Z. Fan, "Damage detection in quasiisotropic composite bends using ultrasonic feature guided waves," Composites Science and Technology, vol. 141, pp. 120-129, 2017.

[10] Y. Zhao, F. Li, P. Cao et al., "Generation mechanism of nonlinear ultrasonic Lamb waves in thin plates with randomly distributed micro-cracks," Ultrasonics, vol. 79, pp. 60-67, 2017. 
[11] B. Masserey and P. Fromme, "Analysis of high frequency guided wave scattering at a fastener hole with a view to fatigue crack detection," Ultrasonics, vol. 76, pp. 78-86, 2017.

[12] P. Huthwaite and F. Simonetti, "High-resolution guided wave tomography," Wave Motion, vol. 50, no. 5, pp. 979-993, 2013.

[13] M. Y. Bhuiyan, Y. Shen, and V. Giurgiutiu, "Guidedwave based crack detection in the rivet hole using global analytical with local FEM approach," Materials, vol. 9, no. 7, article no. 602, 2016.

[14] J. Chen, S. Yuan, L. Qiu, J. Cai, and W. Yang, "Research on a lamb wave and particle filter-based on-line crack propagation prognosis method," Sensors, vol. 16, no. 3, article no. 320, 2016.

[15] J. Cai, S. Yuan, and T. Wang, "Signal construction-based dispersion compensation of lamb waves considering signal waveform and amplitude spectrum preservation," Materials, vol. 10, no. 1, article no. 4, 2017.

[16] Z. Su, C. Yang, N. Pan, L. Ye, and L.-M. Zhou, "Assessment of delamination in composite beams using shear horizontal ( $\mathrm{SH}$ ) wave mode," Composites Science and Technology, vol. 67, no. 2, pp. 244-251, 2007.

[17] P. F. Pai, H. Deng, and M. J. Sundaresan, “Time-frequency characterization of lamb waves for material evaluation and damage inspection of plates," Mechanical Systems and Signal Processing, pp. 183-206, 2015.

[18] V. Giurgiutiu, "Piezoelectric wafer active sensors for structural health monitoring of composite structures using tuned guided waves," Journal of Engineering Materials and Technology, vol. 133, no. 4, Article ID 041012, 2011.

[19] N. E. Huang, Z. Shen, S. R. Long et al., "The empirical mode decomposition and the Hilbert spectrum for nonlinear and non-stationary time series analysis," Proceedings A, vol. 454, no. 1971, pp. 903-995, 1998. 


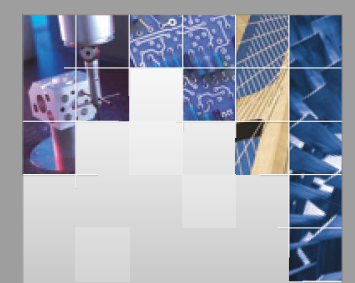

\section{Enfincering}
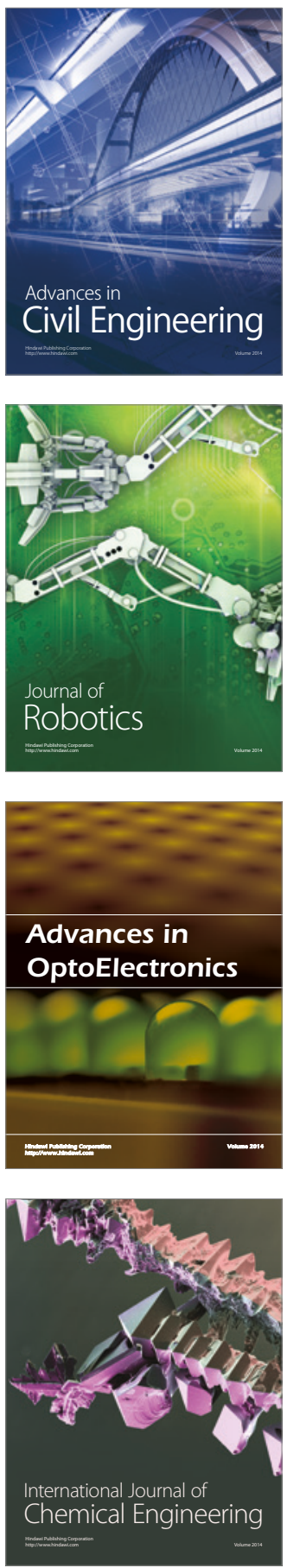

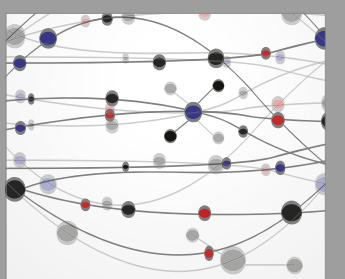

The Scientific World Journal

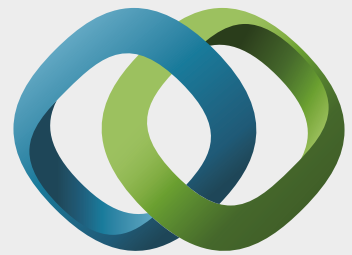

\section{Hindawi}

Submit your manuscripts at

https://www.hindawi.com
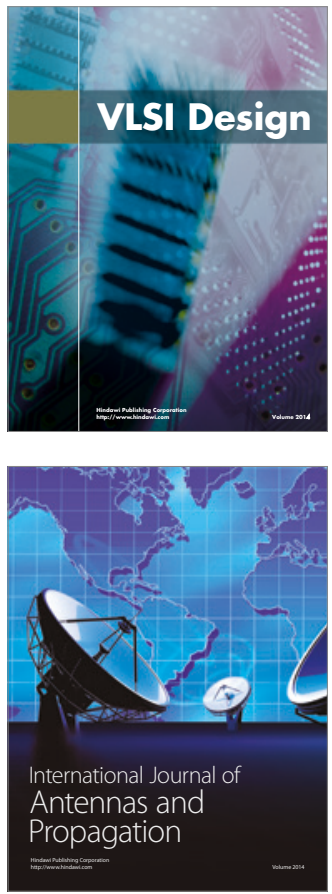

\section{Rotating}

Machinery
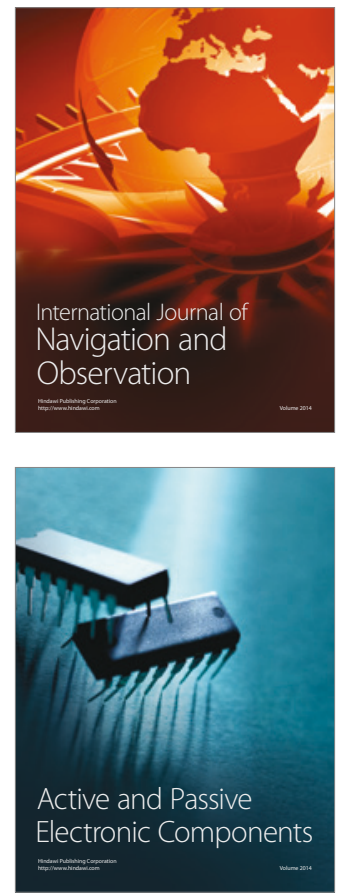
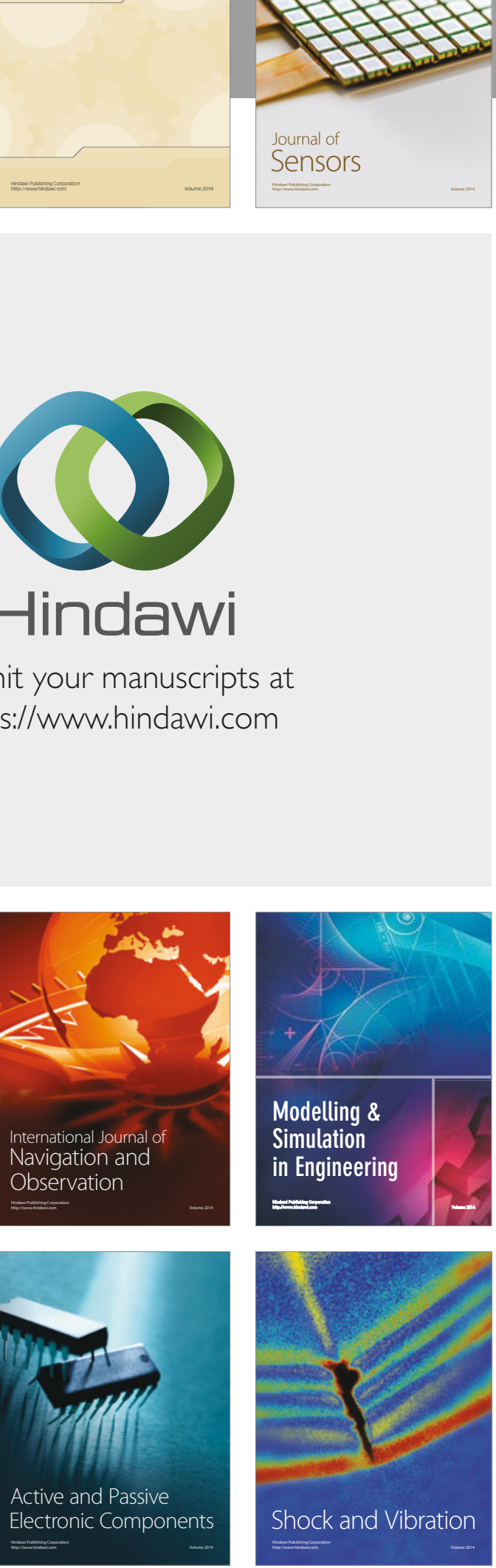
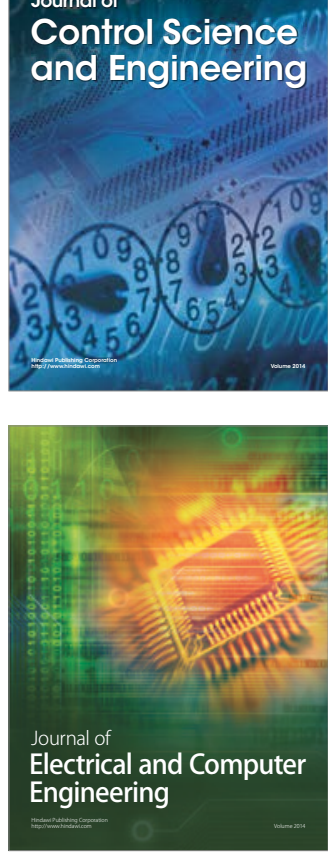

Distributed

Journal of

Control Science

and Engineering
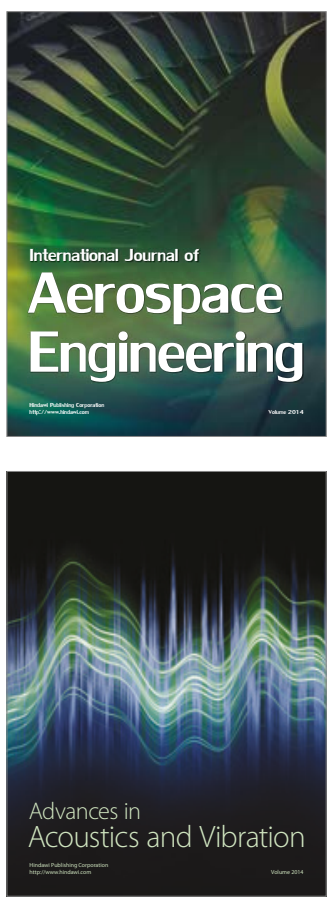

Sensor Networks 